2 Research Square

\title{
Urine di-(2-Ethylhexyl) Phthalate Metabolites Are Independently Related To Body Fluid Status in Adults: Results From a U.S. Nationally Representative Survey
}

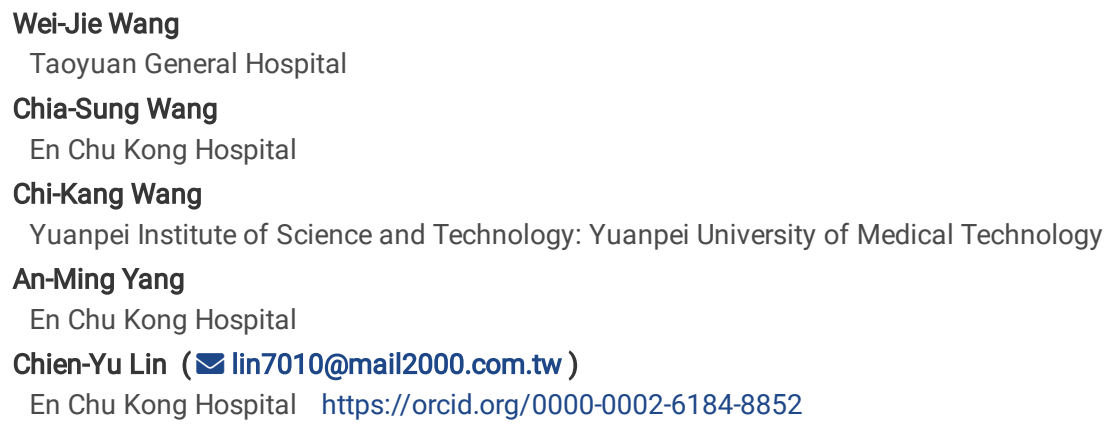




\section{Abstract \\ Background}

Di-(2-ethylhexyl) phthalate (DEHP) has been utilized in many daily used products for decades. Previous studies have reported DEHP exposure could induce renin-angiotensin-aldosterone system activation and increase epithelial sodium channel (ENaC) activity, which contributes to extracellular fluid (ECF) volume expansion. However, there is also no previous study to evaluate the association between DEHP exposure and body fluid status.

\section{Methods}

We selected 1,678 subjects (aged $\geqq 18$ years) from a National Health and Nutrition Examination Survey (NHANES) in 2003-2004 to determine the relationship among urine DEHP metabolites and body composition (body measures, bioelectrical impedance analysis (BIA)).

\section{Results}

After weighted for sampling strategy, we reported higher levels of DEHP metabolites was correlated with increases in body measures (body weight, body mass index (BMI), waist), parameters of BIA (estimated fat mass, percent body fat, ECF, and ECF /intracellular fluid (ICF) ratio) in multiple linear regression analysis. The relationship between DEHP metabolites with ECF/ICF ratio were more evident in subjects with younger age (20-39 year-old), women, non-Hispanic white ethnic, and subjects who were not active smokers.

\section{Conclusions}

Besides positively correlated with body measures and body fat, we found urine DEHP metabolites are positively correlated with ECF, ECF/ICF ratio in the US general adult population. It is necessary to do further research to clarify this causal relationship.

\section{Background}

Phthalates, known as EDCs, are the most common chemicals widely used in many products to increase the flexibility [1, 2]. People are widely exposed to phthalates because its metabolites are commonly detected in urine samples around the world [3]. Although the half-life of phthalates is only about 12 hours [4], due to continuous exposure, their effects are similar to persistent and bioaccumulating compounds [5]. Among the compound esters of phthalates, di-(2ethylhexyl) phthalate (DEHP) has been the main plasticizer used for decades [6]. Recently, several epidemiological studies have linked DEHP exposure to several cardiovascular disease risk factors, such as diabetes mellitus [7] and higher systolic blood pressure [8].

In healthy adults, extracellular fluid (ECF) content composed $~ 33-40 \%$ of the total body water [9] and is regulated by the activity of the renin-angiotensinaldosterone system (RAAS), autonomic nervous system, hormones, and natriuretic peptides [10]. The increased ECF might cause stress in cardiovascular system, which eventually contributes to the hypertension [11] and have been reported as an independent predictor of cardiovascular morbidity in patients with chronic renal failure [12]. The ECF/intracellular fluid (ICF) volume ratio also shows a significant association with chronic disease [13-16]. Recently, many environmental chemicals were reported to have influence on blood pressure and ECF. The mechanism was related to activation of RAAS by inhibiting $11 \beta$ hydroxysteroid dehydrogenase, type 2 (11ß-HSD-2) enzyme activity [17]. The activation of RAAS contributes to sodium retention and ECF expansion [18]. Previous animal and human studies have reported DEHP exposure were associated activation of RAAS through inhibiting 11ß-HSD-2 [19, 20]. Moreover, DEHP exposure might increase the risk of obesity [21], while adipocytes are an important source of extra-adrenal aldosterone [22]. In addition, DEHP have been reported to increase epithelial sodium channel $(\mathrm{ENaC})$ activity in renal cortical collecting duct cells [20], which can expand ECF volume in turn [23]. However, there is no previous epidemiological study to investigate the relationship between DEHP exposure and fluid status.

Bioimpedance analysis (BIA) is a tool for measuring electrical impedance of human tissues, which can be used to assess the proportion of lean mass, fat, and body fluids [24]. Unlike fluid volume, the association between DEHP exposure and other body measures has been extensively studied. When using body mass index (BMI), weight, and waist as parameters, some results showed higher levels of urine DEHP metabolites showed positive correlation [25-27] while others showed no correlation [28], or an inverse association [29]. The relationship between DEHP exposure and body fat percentage has been most investigated in children, and the results were inconsistent [30-33]. In adults, however, there were only few studies studying the association between DEHP exposure and lean mass/body fat percentage and the results were also inconsistent. [34, 35].

Since the relationship DEHP exposure and fluid status is unknown and there were few studies investigated the association between DEHP and lean mass/fat percentage in adults, to resolve the above questions, we included subjects enrolled by National Health and Nutrition Examination Survey (NHANES) 2003 to 2004, who have detailed information on urine DEHP metabolites, body measures, BIA, and various covariates. We hypothesized that urine DEHP metabolites may have a positive correlation with body measures, lean mass, fat mass, and body fluids in this representative sample of U.S. adults.

\section{Materials And Methods}

\section{Study design and population:}


The data for this study was obtained from NHANES 2003-2004. NHANES is a study of a representative sample of the U.S. population that collects information about family health and nutrition. The survey data is released every two years. Detailed contents of the NHANES 2003-2004 are available at the NHANES website [36]. Our analysis included 1,678 participants over the age of 18 without missing data on basic demographics, and BMI, and those who had undergone urine DEHP metabolite testing.

\section{Urine DEHP metabolites:}

In NHANES 2003-2004, urine DEHP metabolites including mono (2-ethylhexyl) phthalate (MEHP), mono(2-ethyl-5-oxohexyl) phthalate (MEOHP), mono(2ethyl-5-hydroxyhexyl) phthalate (MEHHP), and mono(2-ethyl-5-carboxypentyl) phthalate (MECPP) were tested by using high performance liquid chromatography-electrospray ionization-tandem mass spectrometry. For concentrations below detection limits, a value was assigned by NHANES. We used this value in our analyses. The detailed method is available at supplemental section.

\section{Body Measures:}

The body measurement assessments performed on survey participants varied according to the participants' ages. Weight, BMI, triceps and subscapular skinfold were measured in NHANES 2003-2004. The detailed method is available at supplemental section.

\section{Bioelectrical Impedance Analysis (BIA):}

In NHANES 2003-2004, this examination was conducted in eligible survey participants 8-49 years of age. The BIA data were collected with a HYDRA ECF/ICF Bio-Impedance Spectrum Analyzer (Model 4200, Xitron Technologies, Inc., San Diego, California, USA). Detailed information is available at the supplemental section.

\section{Covariates:}

We considered age, gender, race/ethnicity, education level, household income, smoking status, caffeine intake, total energy intake, total protein intake, total sugar intake, total carbohydrate intake, total saturated fatty acid intake, metabolic equivalent intensity level for activity to be potential confounders in this study. Detailed information is available at the supplemental section.

\section{Statistics}

DEHP metabolites concentrations were corrected for urine creatinine and expressed as the geometric mean (geometric standard error) in different subpopulations. These variables were tested by Student's 2-tailed t-test and one-way analysis of variance. Because DEHP is metabolized primarily into MEHP, MEOHP, MEHHP, and MECPP, we divided the level of each metabolite by its molar mass, and then added the concentrations of each metabolite to calculate $\Sigma$ DEHP [37]. Due to the obvious deviation from the normal distribution, the DEHP metabolites were transformed by natural logarithm. We constructed an extended model approach with body measures/ bioelectrical impedance analysis as the dependent variable and individual In-DEHP metabolites as a predictor. Model 1 adjusted for age, gender, race and ethnicity, education level, household income, smoking status, and metabolic equivalent intensity level for activity. Model 2 adjusted for model 1 plus caffeine intake, total energy intake, total protein intake, total sugar intake, total carbohydrate intake, total saturated fatty acid intake.

We used sample weights for analysis to understand the impact of weights. The calculation of sampling weight follows the analysis guidelines of the National Center for Health Statistics, and appropriately considers the complex survey design adopted in NHANES 2003-2004 [38]. All analyses were calculated by SPSS Version 20 (SPSS Inc. Chicago, Illinois, U.S.A.). $P<0.05$ was considered significant.

\section{Results}

MEHP, MEOHP, MEHHP, and MECPP were detectable in $69.9 \%, 99.1 \%, 99.7 \%$, and $100 \%$ of study subjects, respectively. Table 1 shows the basic demographics of study subjects. The study participants composed of 798 men and 880 women. Subjects with aged between $40-59$ years old, women, and higher education level were associated with higher all DEHP metabolites concentrations and $\Sigma$ DEHP. Besides MEHP, subjects with higher income were associated with higher levels of other DEHP metabolites and $\Sigma$ DEHP while these metabolites levels were different between races. Participants with BMI between $25-30$ had a lower concentration of DEHP metabolites and $\Sigma$ DEHP while active smokers had a lower concentration of MEOHP, MECPP, and $\Sigma$ DEHP. Those who had body fat percentage $\geq 35 \%$ had a higher level of MEOHP, MECPP, and $\Sigma$ DEHP. 
Table 1

Basic demographics of the sample subjects including geometric means (geometric S.E.) of DEHP metabolites concentrations

\begin{tabular}{|c|c|c|c|c|c|c|}
\hline & Unweighted no. (\%) & MEHP $(\mu \mathrm{g} / \mathrm{g})$ & MEOHP $(\mu \mathrm{g} / \mathrm{g})$ & MEHHP $(\mu \mathrm{g} / \mathrm{g})$ & $\begin{array}{l}\text { MECPP } \\
(\mu \mathrm{g} / \mathrm{g})\end{array}$ & $\begin{array}{l}\Sigma \text { DEHP } \\
(\mu \mathrm{mol} / \mathrm{g})\end{array}$ \\
\hline Overall & $1678(100)$ & $2.13(1.03)$ & $12.18(1.03)$ & $18.23(1.03)$ & $29.44(1.03)$ & $0.21(1.03)$ \\
\hline \multicolumn{7}{|l|}{ Age, y } \\
\hline $18-39$ & $718(42.8)$ & $2.64(1.05)^{\ddagger}$ & $13.63(1.05)^{\ddagger}$ & $20.15(1.05)^{\dagger}$ & $30.88(1.04)^{\dagger}$ & $0.23(1.04)^{\dagger}$ \\
\hline $40-59$ & $449(26.8)$ & $1.90(1.06)^{\ddagger}$ & $10.67(1.05)^{\ddagger}$ & $16.33(1.05)^{\dagger}$ & $25.89(1.05)^{\dagger}$ & $0.19(1.05)^{\dagger}$ \\
\hline$\geq 60$ & $511(30.4)$ & $1.72(1.05)^{\ddagger}$ & $11.70(1.04)^{\ddagger}$ & $17.46(1.04)^{\dagger}$ & $30.81(1.04)^{\dagger}$ & $0.21(1.04)^{\dagger}$ \\
\hline \multicolumn{7}{|l|}{ Gender } \\
\hline Men & $798(47.6)$ & $1.85(1.05)^{\ddagger}$ & $10.74(1.04)^{\ddagger}$ & $16.52(1.04)^{\ddagger}$ & $26.08(1.04)^{\ddagger}$ & $0.19(1.04)^{\ddagger}$ \\
\hline Women & $880(52.4)$ & $2.41(1.04)^{\ddagger}$ & $13.66(1.04)^{\ddagger}$ & $19.94(1.04)^{\ddagger}$ & $32.86(1.03)^{\ddagger}$ & $0.24(1.03)^{\ddagger}$ \\
\hline \multicolumn{7}{|l|}{ Race } \\
\hline Mexican American & $365(21.8)$ & $1.96(1.06)$ & $10.36(1.06)^{\star}$ & $15.18(1.06)^{\star}$ & $26.30(1.05)^{\star}$ & $0.19(1.05)^{\star}$ \\
\hline Other Hispanic & 48 (2.9) & $2.63(1.17)$ & $12.92(1.17)^{\star}$ & $19.59(1.18)^{\star}$ & $32.47(1.14)^{*}$ & $0.24(1.15)^{\star}$ \\
\hline Non-Hispanic White & $842(50.2)$ & $2.09(1.04)$ & $12.91(1.04)^{\star}$ & $19.19(1.04)^{\star}$ & $32.00(1.04)^{\star}$ & $0.23(1.04)^{\star}$ \\
\hline Non-Hispanic Black & $345(20.6)$ & $2.37(1.07)$ & $12.72(1.06)^{\star}$ & $19.64(1.06)^{\star}$ & $26.91(1.06)^{\star}$ & $0.21(1.06)^{\star}$ \\
\hline Others & $78(4.5)$ & $2.02(1.14)$ & $11.09(1.13)^{\star}$ & $17.09(1.13)^{\star}$ & $28.41(1.12)^{\star}$ & $0.20(1.12)^{\star}$ \\
\hline \multicolumn{7}{|l|}{ Education levels } \\
\hline$\leqq$ High school & $927(55.3)$ & $1.94(1.04)^{\ddagger}$ & $10.85(1.03)^{\ddagger}$ & $16.04(1.04)^{\ddagger}$ & $26.54(1.03)^{\ddagger}$ & $0.19(1.03)^{\ddagger}$ \\
\hline >High school & $750(44.7)$ & $2.37(1.05)^{\ddagger}$ & $14.06(1.04)^{\ddagger}$ & $21.35(1.04)^{\ddagger}$ & $33.47(1.04)^{\ddagger}$ & $0.25(1.04)^{\ddagger}$ \\
\hline \multicolumn{7}{|c|}{ Annual household income } \\
\hline$<\$ 25000$ & $556(35.6)$ & $2.02(1.05)$ & $10.98(1.05)^{\ddagger}$ & $16.50(1.05)^{\ddagger}$ & $27.52(1.04)^{\dagger}$ & $0.20(1.04)^{\ddagger}$ \\
\hline$\$ 25000-55000$ & $503(32.2)$ & $2.00(1.05)$ & $11.54(1.05)^{\ddagger}$ & $17.24(1.05)^{\ddagger}$ & $27.89(1.05)^{\dagger}$ & $0.20(1.05)^{\ddagger}$ \\
\hline$>\$ 55000$ & $501(32.2)$ & $2.31(1.06)$ & $14.07(1.05)^{\ddagger}$ & $21.06(1.05)^{\ddagger}$ & $33.01(1.05)^{\dagger}$ & $0.24(1.05)^{\ddagger}$ \\
\hline \multicolumn{7}{|l|}{$\mathrm{BMI}, \mathrm{kg} / \mathrm{m}^{2}$} \\
\hline$<25$ & $577(34.4)$ & $2.31(1.05)^{\star}$ & $11.92(1.05)^{\ddagger}$ & $17.77(1.05)^{\ddagger}$ & $28.71(1.05)^{*}$ & $0.21(1.05)^{\ddagger}$ \\
\hline $25-30$ & $562(33.5)$ & $1.90(1.05)^{\star}$ & $10.93(1.05)^{\ddagger}$ & $16.31(1.05)^{\ddagger}$ & $27.29(1.04)^{\star}$ & $0.20(1.04)^{\ddagger}$ \\
\hline$\geq 30$ & $539(32.1)$ & $2.19(1.05)^{\star}$ & $13.97(1.05)^{\ddagger}$ & $21.06(1.05)^{\ddagger}$ & $32.73(1.04)^{*}$ & $0.24(1.04)^{\ddagger}$ \\
\hline \multicolumn{7}{|c|}{ Body fat percentage (\%) } \\
\hline$<25$ & $227(31.4)$ & $2.22(1.09)$ & $10.70(1.08)^{*}$ & $16.81(1.09)$ & $25.82(1.08)^{\star}$ & $0.19(1.08)^{\star}$ \\
\hline $25-35$ & $230(31.9)$ & $2.00(1.09)$ & $11.33(1.08)^{*}$ & $17.11(1.08)$ & $25.87(1.07)^{\star}$ & $0.20(1.07)^{\star}$ \\
\hline$\geq 35$ & $265(36.7)$ & $2.53(1.08)$ & $14.19(1.07)^{\star}$ & $20.83(1.08)$ & $32.21(1.07)^{\star}$ & $0.24(1.07)^{\star}$ \\
\hline \multicolumn{7}{|l|}{ Smoking } \\
\hline Nonexposed & $353(21.0)$ & $2.16(1.07)$ & $12.10(1.06)^{\star}$ & $18.26(1.06)$ & $31.27(1.05)^{\ddagger}$ & $0.22(1.06)^{\star}$ \\
\hline Expose to ETS & $849(50.6)$ & $2.15(1.04)$ & $12.99(1.04)^{\star}$ & $19.04(1.04)$ & $31.15(1.04)^{\ddagger}$ & $0.23(1.04)^{\star}$ \\
\hline Active smokers & $476(28.4)$ & $2.07(1.06)$ & $10.93(1.05)^{\star}$ & $18.23(1.05)$ & $25.46(1.05)^{\ddagger}$ & $0.19(1.05)^{\star}$ \\
\hline
\end{tabular}

$*, p<0.05 ;{ }^{\dagger}, p<0.01 ;{ }^{\ddagger}, p<0.005$ (tested by Student's 2-tailed t-test or by one-way analysis of variance)

Abbreviations: DEHP, di-(2-ethylhexyl) phthalate; ETS. Environmental tobacco smoke; MEHP, mono(2-ethylhexyl) phthalate; MEHHP, mono(2-ethyl-5hydroxyhexyl) phthalate; MEOHP, mono(2-ethyl-5-oxohexyl) phthalate; MECPP, Mono(2-ethyl-5-carboxypentyl) phthalate.

$\Sigma$ DEHP were the sum of $(\mathrm{MEHP} / 278)+(\mathrm{MEHHP} / 294)+(\mathrm{MEOHP} / 292)+(\mathrm{MECPP} / 308)$ and corrected for urine creatinine 


\begin{tabular}{|c|c|c|c|c|c|c|}
\hline & Unweighted no. (\%) & MEHP $(\mu \mathrm{g} / \mathrm{g})$ & MEOHP $(\mu \mathrm{g} / \mathrm{g})$ & MEHHP $(\mu \mathrm{g} / \mathrm{g})$ & $\begin{array}{l}\text { MECPP } \\
(\mu \mathrm{g} / \mathrm{g})\end{array}$ & $\begin{array}{l}\Sigma \mathrm{DEHP} \\
(\mu \mathrm{mol} / \mathrm{g})\end{array}$ \\
\hline$<12$ & $412(29.5)$ & $2.01(1.06)$ & $11.86(1.05)$ & $17.38(1.05)$ & $2.52(1.05)$ & $0.21(1.05)$ \\
\hline$\geq 12$ & $983(70.5)$ & $2.05(1.04)$ & $11.89(1.04)$ & $17.97(1.04)$ & $2.32(1.03)$ & $0.21(1.03)$ \\
\hline
\end{tabular}

$*, p<0.05 ;{ }^{\dagger}, p<0.01 ;{ }^{\ddagger}, p<0.005$ (tested by Student's 2-tailed t-test or by one-way analysis of variance)

Abbreviations: DEHP, di-(2-ethylhexyl) phthalate; ETS. Environmental tobacco smoke; MEHP, mono(2-ethylhexyl) phthalate; MEHHP, mono(2-ethyl-5hydroxyhexyl) phthalate; MEOHP, mono(2-ethyl-5-oxohexyl) phthalate; MECPP, Mono(2-ethyl-5-carboxypentyl) phthalate.

$\Sigma$ DEHP were the sum of $(\mathrm{MEHP} / 278)+(\mathrm{MEHHP} / 294)+(\mathrm{MEOHP} / 292)+(\mathrm{MECPP} / 308)$ and corrected for urine creatinine

The means and S.E. of body fluid status between basic demographic of the sample subjects are shown in Table 2. Subjects with men, higher household income, higher BMI, lower body fat percentage, active smoking, and higher alcohol consumption were associated with higher ECF and ICF volume. Higher ECF volume was also found in subjects who were non-Hispanic white. Subjects with aged between 40-59 years old, women, higher education level, lower BMI, higher body fat percentage, and lower alcohol consumption were associated with higher ECF/ICF ration while lower ECF/ICF ratio was found in other Hispanic. 
Table 2

Basic demographics of the sample subjects including means (S.E.) of body fluid status

\begin{tabular}{|c|c|c|c|c|}
\hline & \multirow[t]{2}{*}{ Unweighted no. (\%) } & \multirow{2}{*}{$\begin{array}{l}\text { ECF } \\
\text { (L) }\end{array}$} & \multirow{2}{*}{$\begin{array}{l}\text { ICF } \\
\text { (L) }\end{array}$} & \multirow[t]{2}{*}{ ECF/ICF ratio } \\
\hline & & & & \\
\hline Overall & $726(100)$ & $16.92(0.13)$ & $23.35(0.24)$ & $0.74(0.00)$ \\
\hline \multicolumn{5}{|l|}{ Age, y } \\
\hline $18-39$ & $524(42.8)$ & $16.80(0.16)$ & $23.42(0.29)$ & $0.74(0.00)^{\ddagger}$ \\
\hline $40-50$ & $202(26.8)$ & $17.24(0.24)$ & $23.16(0.42)$ & $0.76(0.01)^{\ddagger}$ \\
\hline \multicolumn{5}{|l|}{ Gender } \\
\hline Men & $377(47.6)$ & $19.00(0.15)^{\ddagger}$ & $27.61(0.28)^{\ddagger}$ & $0.70(0.00)^{\ddagger}$ \\
\hline Women & $349(52.4)$ & $14.67(0.15)^{\ddagger}$ & $18.74(0.22)^{\ddagger}$ & $0.79(0.00)^{\ddagger}$ \\
\hline \multicolumn{5}{|l|}{ Race } \\
\hline Mexican American & $172(21.8)$ & $15.95(0.23)^{\ddagger}$ & $22.32(0.43)$ & $0.73(0.01)^{\star}$ \\
\hline Other Hispanic & $27(2.9)$ & $15.99(0.64)^{\ddagger}$ & $22.92(1.28)$ & $0.72(0.02)^{\star}$ \\
\hline Non-Hispanic White & $319(50.2)$ & $17.45(0.21)^{\ddagger}$ & $23.78(0.38)$ & $0.75(0.01)^{\star}$ \\
\hline Non-Hispanic Black & $172(20.6)$ & $17.13(0.27)^{\ddagger}$ & $23.78(0.49)$ & $0.74(0.01)^{\star}$ \\
\hline Others & $36(4.5)$ & $16.59(0.65)^{\ddagger}$ & $22.70(1.22)$ & $0.75(0.01)^{\star}$ \\
\hline \multicolumn{5}{|l|}{ Education levels } \\
\hline$\leqq$ High school & $376(55.3)$ & $16.88(0.18)$ & $23.58(0.33)$ & $0.73(0.00)^{\ddagger}$ \\
\hline$>$ High school & $350(44.7)$ & $16.97(0.20)$ & $23.10(0.36)$ & $0.75(0.00)^{\ddagger}$ \\
\hline \multicolumn{5}{|c|}{ Annual household income } \\
\hline$<\$ 25000$ & $203(35.6)$ & $16.40(0.23)^{\ddagger}$ & $22.50(0.41)^{\star}$ & $0.74(0.01)$ \\
\hline$\$ 25000-55000$ & $227(32.2)$ & $16.79(0.24)^{\ddagger}$ & $23.32(0.44)^{*}$ & $0.74(0.01)$ \\
\hline$>\$ 55000$ & $248(32.2)$ & $17.62(0.24)^{\ddagger}$ & $24.25(0.45)^{\star}$ & $0.75(0.00)$ \\
\hline \multicolumn{5}{|l|}{$\mathrm{BMI}, \mathrm{kg} / \mathrm{m}^{2}$} \\
\hline$<25$ & $301(34.4)$ & $15.04(0.18)^{\ddagger}$ & $20.04(0.31)^{\ddagger}$ & $0.77(0.01)^{\ddagger}$ \\
\hline $25-30$ & $218(33.5)$ & $17.22(0.20)^{\ddagger}$ & $24.35(0.38)^{\ddagger}$ & $0.72(0.01)^{\ddagger}$ \\
\hline$\geq 30$ & $207(32.1)$ & $19.35(0.23)^{\ddagger}$ & $27.10(0.46)^{\ddagger}$ & $0.73(0.01)^{\ddagger}$ \\
\hline \multicolumn{5}{|c|}{ Body fat percentage (\%) } \\
\hline$<25$ & $229(31.4)$ & $18.52(0.21)^{\ddagger}$ & $27.79(0.38)^{\ddagger}$ & $0.67(0.00)^{\ddagger}$ \\
\hline $25-35$ & 230 (31.9) & $16.94(0.26)^{\ddagger}$ & $23.24(0.42)^{\ddagger}$ & $0.74(0.00)^{\ddagger}$ \\
\hline$\geq 35$ & $267(36.7)$ & $15.54(0.19)^{\ddagger}$ & $19.62(0.29)^{\ddagger}$ & $0.80(0.01)^{\ddagger}$ \\
\hline \multicolumn{5}{|l|}{ Smoking } \\
\hline Nonexposed & $109(21.0)$ & $15.91(0.35)^{\ddagger}$ & $21.45(0.60)^{\ddagger}$ & $0.76(0.01)$ \\
\hline Expose to ETS & $356(50.6)$ & $16.74(0.19)^{\ddagger}$ & $23.23(0.35)^{\ddagger}$ & $0.74(0.00)$ \\
\hline Active smokers & $257(28.4)$ & $17.63(0.21)^{\ddagger}$ & $24.39(0.39)^{\ddagger}$ & $0.74(0.01)$ \\
\hline \multicolumn{5}{|c|}{ Alcohol consumption (drink/year) } \\
\hline$<12$ & $124(29.5)$ & $16.34(0.36)^{\ddagger}$ & b21.87(0.62) $)^{\ddagger}$ & $0.77(0.01)^{\ddagger}$ \\
\hline
\end{tabular}

${ }^{*}, \mathrm{p}<0.05 ;{ }^{\dagger}, \mathrm{p}<0.01 ;{ }^{\ddagger}, \mathrm{p}<0.005$ (tested by Student's 2-tailed t-test or by one-way analysis of variance)

Abbreviations: ETS. Environmental tobacco smoke; 


\begin{tabular}{|lclll|}
\hline & Unweighted no. (\%) & ECF & ICF & ECF/ICF ratio \\
& & $(\mathrm{L})$ & $(\mathrm{L})$ & \\
\hline$\geq 12$ & $429(70.5)$ & $17.43(0.17)^{\ddagger}$ & $24.23(0.31)^{\ddagger}$ & $0.74(0.00)^{\ddagger}$ \\
\hline${ }^{*}, \mathrm{p}<0.05 ;{ }^{\dagger}, \mathrm{p}<0.01 ;{ }^{\ddagger}, \mathrm{p}<0.005$ (tested by Student's 2-tailed t-test or by one-way analysis of variance) \\
\hline Abbreviations: ETS. Environmental tobacco smoke; & & \\
\hline
\end{tabular}

After weighting for sampling strategy, the correlations between DEHP metabolite levels and various body component measures are shown in Table 3. Levels of MEOHP, MEHHP, MECPP, and LDEHP was positively associated with weight, BMI, waist. The correlations between DEHP metabolite levels and parameters of bioelectrical impedance analysis are shown in Table 4. Levels of MEOHP, MEHHP, MECPP, and $\Sigma$ DEHP was positively correlated with estimated fat mass, estimated percent body fat, ECF, and ECF/ICF ratio.

Table 3

Linear regression coefficients (S.E.) of body measures with one unit increase in In-DEHP metabolites concentrations in adults, with results weighted for sam

\begin{tabular}{|c|c|c|c|c|c|c|c|c|c|c|}
\hline $\begin{array}{l}\text { Body } \\
\text { measures }\end{array}$ & & $\begin{array}{l}\text { MEHP } \\
(\mu g / g)\end{array}$ & $P$ & $\begin{array}{l}\text { MEOHP } \\
(\mu \mathrm{g} / \mathrm{g})\end{array}$ & $P$ & $\begin{array}{l}\text { MEHHP } \\
(\mu \mathrm{g} / \mathrm{g})\end{array}$ & $P$ & $\begin{array}{l}\text { MECPP } \\
(\mu \mathrm{g} / \mathrm{g})\end{array}$ & $P$ & $\begin{array}{l}\Sigma \text { DEHP } \\
(\mu \mathrm{mol} / \mathrm{g})\end{array}$ \\
\hline \multicolumn{11}{|c|}{$\begin{array}{l}\text { Body weight } \\
\text { (Kg) }\end{array}$} \\
\hline Model 1 & $1563 / 193092305$ & $0.092(0.501)$ & 0.856 & $2.020(0.760)$ & 0.018 & $2.047(0.648)$ & 0.007 & $2.003(0.766)$ & 0.019 & $2.031(0.749)$ \\
\hline Model 2 & $852 / 117709513$ & $0.023(0.452)$ & 0.961 & $1.679(0.572)$ & 0.010 & $1.704(0.538)$ & 0.006 & $1.520(0.616)$ & 0.026 & $1.627(0.593)$ \\
\hline \multicolumn{11}{|c|}{$\begin{array}{l}\text { Body mass } \\
\text { index } \\
\left(\mathrm{kg} / \mathrm{m}^{2}\right)\end{array}$} \\
\hline Model 1 & $1563 / 193092305$ & $0.060(0.157)$ & 0.711 & $0.679(0.211)$ & 0.006 & $0.696(0.190)$ & 0.002 & $0.709(0.227)$ & 0.007 & $0.705(0.217)$ \\
\hline Model 2 & $852 / 117709513$ & $0.052(0.145)$ & 0.726 & $0.598(0.174)$ & 0.004 & $0.617(0.177)$ & 0.003 & $0.578(0.200)$ & 0.011 & $0.602(0.189)$ \\
\hline \multicolumn{11}{|l|}{ Waist (cm) } \\
\hline Model 1 & $1563 / 193092305$ & $-0.012(0.409)$ & 0.978 & $1.549(0.534)$ & 0.011 & $1.532(0.468)$ & 0.005 & $1.512(0.515)$ & 0.010 & $1.533(0.521)$ \\
\hline Model 2 & $852 / 117709513$ & $0.059(0.414)$ & 0.888 & $1.374(0.423)$ & 0.005 & $1.342(0.403)$ & 0.005 & $1.209(0.391)$ & 0.007 & $1.305(0.411)$ \\
\hline \multicolumn{11}{|c|}{$\begin{array}{l}\text { Subscapular } \\
\text { Skinfold } \\
(\mathrm{mm})\end{array}$} \\
\hline Model 1 & $1255 / 1158342664$ & $-0.362(0.210)$ & 0.104 & $0.082(0.274)$ & 0.770 & $0.151(0.272)$ & 0.586 & $0.133(0.278)$ & 0.640 & $0.073(0.284)$ \\
\hline Model 2 & $687 / 97486158$ & $-0.423(0.206)$ & 0.058 & $0.019(0.258)$ & 0.943 & $0.113(0.258)$ & 0.669 & $0.038(0.244)$ & 0.877 & $0.005(0.260)$ \\
\hline \multicolumn{11}{|l|}{$\begin{array}{l}\text { Triceps } \\
\text { Skinfold } \\
(\mathrm{mm})\end{array}$} \\
\hline Model 1 & $1390 / 172886004$ & $-0.253(0.180)$ & 0.181 & $0.263(0.228)$ & 0.267 & $0.237(0.199)$ & 0.254 & $0.261(0.256)$ & 0.323 & $0.237(0.241)$ \\
\hline Model 2 & $763 / 106411256$ & $-0.198(0.164)$ & 0.245 & $0.272(0.216)$ & 0.227 & $0.234(0.200)$ & 0.262 & $0.205(0.234)$ & 0.395 & $0.216(0.223)$ \\
\hline
\end{tabular}

Model 1 was adjusted for age, gender, race/ethnicity, educational level, household income, smoking status, and metabolic equivalent intensity level for activit Model 2 was adjusted for model 1 plus caffeine intake, total energy intake, total protein intake, total sugar intake, total carbohydrates intake, total saturated $\mathrm{f}$ acids intake,

Abbreviations: DEHP, di-(2-ethylhexyl) phthalate; MEHP, mono(2-ethylhexyl) phthalate; MEHHP, mono(2-ethyl-5-hydroxyhexyl) phthalate; MEOHP, mono(2-ethyl oxohexyl) phthalate; MECPP, Mono(2-ethyl-5-carboxypentyl) phthalate.

$\Sigma$ DEHP were the sum of $(\mathrm{MEHP} / 278)+(\mathrm{MEHHP} / 294)+(\mathrm{MEOHP} / 292)+(\mathrm{MECPP} / 308)$ and corrected for urine creatinine 
Table 4

Linear regression coefficients (S.E.) of bioelectrical impedance analysis parameters with one unit increase in In-DEHP metabolites concentrations in adults, $v$ results weighted for sampling strategy

\begin{tabular}{|c|c|c|c|c|c|c|c|c|c|c|c|}
\hline $\begin{array}{l}\text { Bioelectrical } \\
\text { Impedance } \\
\text { Analysis }\end{array}$ & & $\begin{array}{l}\text { MEHP } \\
(\mu g / g)\end{array}$ & $P$ & $\begin{array}{l}\text { MEOHP } \\
(\mu g / g)\end{array}$ & $P$ & $\begin{array}{l}\text { MEHHP } \\
(\mu g / g)\end{array}$ & $P$ & $\begin{array}{l}\text { MECPP } \\
(\mu \mathrm{g} / \mathrm{g})\end{array}$ & $P$ & $\begin{array}{l}\Sigma \mathrm{DEHP} \\
(\mu \mathrm{mol} / \mathrm{g})\end{array}$ & $F$ \\
\hline \multicolumn{12}{|l|}{$\begin{array}{l}\text { Lean mass } \\
(\mathrm{kg})\end{array}$} \\
\hline Model 1 & $674 / 96499431$ & $-0.012(0.320)$ & 0.970 & $0.654(0.444)$ & 0.162 & $0.692(0.382)$ & 0.090 & $0.562(0.481)$ & 0.262 & $0.635(0.444)$ & 0 \\
\hline Model 2 & $415 / 61088209$ & $-0.020(0.296)$ & 0.948 & $0.608(0.384)$ & 0.134 & $0.640(0.364)$ & 0.099 & $0.497(0.416)$ & 0.251 & $0.579(0.393)$ & 0 \\
\hline \multicolumn{12}{|l|}{$\begin{array}{l}\text { Fat mass } \\
(\mathrm{kg})\end{array}$} \\
\hline Model 1 & $674 / 96499431$ & $0.806(0.610)$ & 0.206 & $1.646(0.675)$ & 0.028 & $1.579(0.617)$ & 0.022 & $1.639(0.697)$ & 0.033 & $1.666(0.695)$ & 0 \\
\hline Model 2 & $415 / 61088209$ & $1.000(0.546)$ & 0.087 & $1.716(0.602)$ & 0.012 & $1.560(0.580)$ & 0.017 & $1.547(0.639)$ & 0.029 & $1.651(0.636)$ & 0 \\
\hline \multicolumn{12}{|l|}{$\begin{array}{l}\text { Percent } \\
\text { body fat (\%) }\end{array}$} \\
\hline Model 1 & $674 / 96499431$ & $0.419(0.377)$ & 0.285 & $0.800(0.383)$ & 0.034 & $0.718(0.368)$ & 0.040 & $0.808(0.374)$ & 0.047 & $0.794(0.391)$ & 0 \\
\hline Model 2 & $415 / 61088209$ & $0.632(0.352)$ & 0.093 & $0.951(0.337)$ & 0.013 & $0.789(0.336)$ & 0.033 & $0.865(0.348)$ & 0.025 & $0.894(0.354)$ & 0 \\
\hline \multicolumn{12}{|l|}{$\begin{array}{l}\text { Cell } \\
\text { membrane } \\
\text { capacitance } \\
(\mathrm{nF})\end{array}$} \\
\hline Model 1 & $674 / 96499431$ & $-0.024(0.028)$ & 0.416 & $-0.008(0.022)$ & 0.716 & $-0.001(0.018)$ & 0.952 & $-0.014(0.021)$ & 0.525 & $-0.009(0.020)$ & 0 \\
\hline Model 2 & 415/61088209 & $-0.022(0.029)$ & 0.469 & $-0.002(0.018)$ & 0.925 & $0.004(0.018)$ & 0.824 & $-0.009(0.020)$ & 0.653 & $-0.004(0.020)$ & 0 \\
\hline \multicolumn{12}{|l|}{$\mathrm{ECF}(\mathrm{L})$} \\
\hline Model 1 & $674 / 96499431$ & $0.134(0.101)$ & 0.203 & $0.321(0.149)$ & 0.049 & $0.308(0.133)$ & 0.035 & $0.316(0.167)$ & 0.078 & $0.327(0.156)$ & 0 \\
\hline Model 2 & $415 / 61088209$ & $0.147(0.085)$ & 0.105 & $0.289(0.126)$ & 0.037 & $0.271(0.119)$ & 0.038 & $0.279(0.143)$ & 0.069 & $0.292(0.134)$ & 0 \\
\hline \multicolumn{12}{|l|}{ ICF (L) } \\
\hline Model 1 & $674 / 96499431$ & $-0.106(0.151)$ & 0.493 & $0.196(0.191)$ & 0.321 & $0.230(0.162)$ & 0.175 & $0.139(0.203)$ & 0.505 & $0.179(0.186)$ & 0 \\
\hline Model 2 & $415 / 61088209$ & $-0.120(0.147)$ & 0.430 & $0.189(0.168)$ & 0.278 & $0.224(0.160)$ & 0.184 & $0.123(0.178)$ & 0.498 & $0.168(0.169)$ & 0 \\
\hline \multicolumn{12}{|l|}{$\begin{array}{l}\mathrm{ECF} / \mathrm{ICF} \\
\text { ratio }\end{array}$} \\
\hline Model 1 & $471 / 68616209$ & $0.008(0.003)$ & 0.010 & $0.008(0.002)$ & 0.004 & $0.006(0.002)$ & 0.021 & $0.009(0.003)$ & 0.005 & $0.008(0.003)$ & 0 \\
\hline Model 2 & $415 / 61088209$ & $0.009(0.003)$ & 0.003 & $0.007(0.002)$ & 0.004 & $0.005(0.002)$ & 0.041 & $0.009(0.002)$ & 0.002 & $0.008(0.002)$ & 0 \\
\hline
\end{tabular}

Model 1 was adjusted for age, gender, race/ethnicity, educational level, household income, smoking status, and metabolic equivalent intensity level for activit Model 2 was adjusted for model 1 plus caffeine intake, total energy intake, total protein intake, total sugar intake, total carbohydrates intake, total saturated $\mathrm{f}$ acids intake,

Abbreviations: DEHP, di-(2-ethylhexyl) phthalate; MEHP, mono(2-ethylhexyl) phthalate; MEHHP, mono(2-ethyl-5-hydroxyhexyl) phthalate; MEOHP, mono(2-ethyl oxohexyl) phthalate; MECPP, Mono(2-ethyl-5-carboxypentyl) phthalate; ECF, extracellular fluid; ICF, intracellular fluid.

$\Sigma$ DEHP were the sum of $(\mathrm{MEHP} / 278)+(\mathrm{MEHHP} / 294)+(\mathrm{MEOHP} / 292)+(\mathrm{MECPP} / 308)$ and corrected for urine creatinine 
Table 5

Linear regression coefficients (SE) between In-LDEHP and body composition parameters in different subpopulations of sample subjects with results weighted for sampling strategy

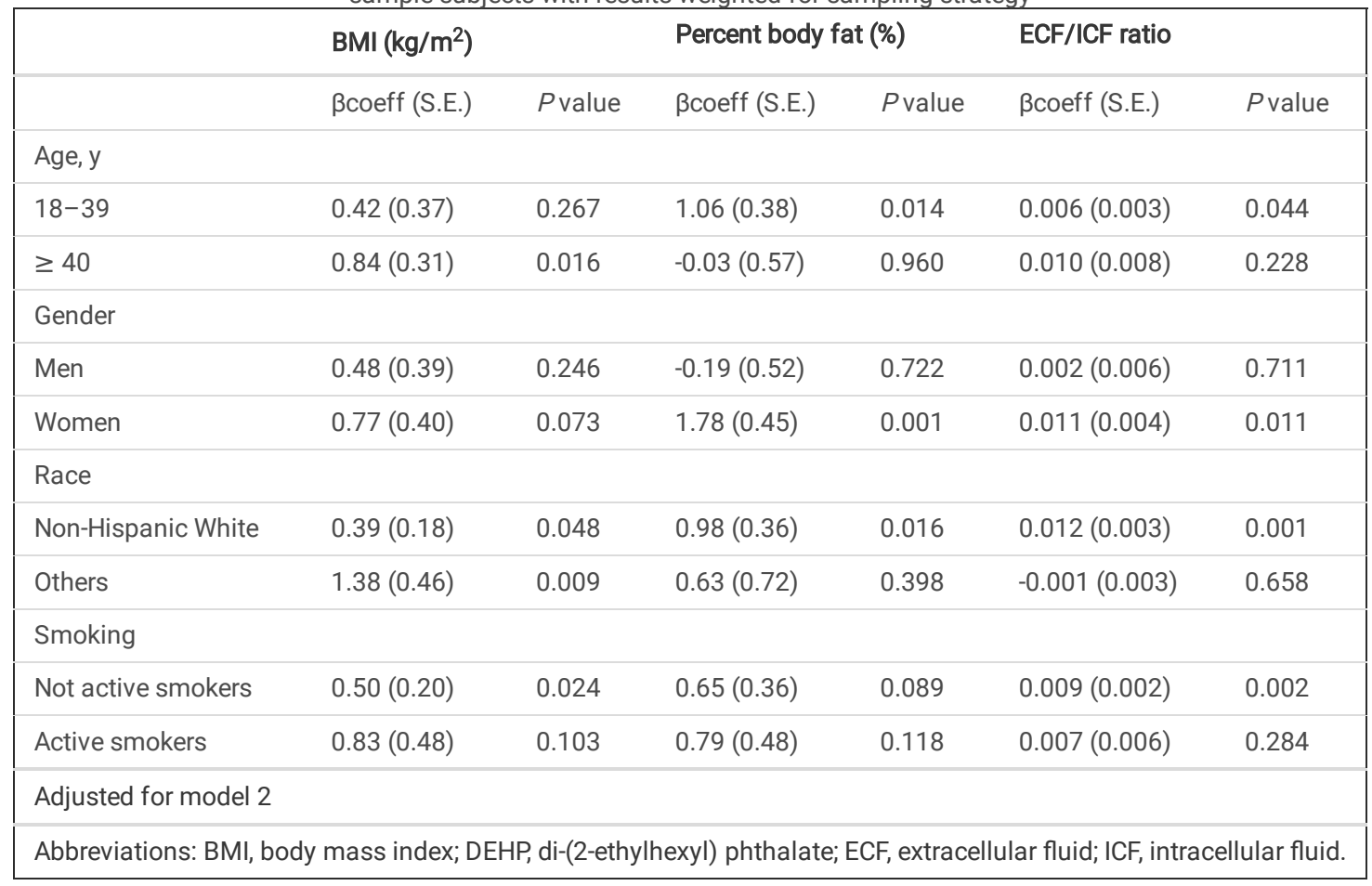

Linear regression coefficients between $\Sigma$ DEHP, BMI, percent fat mass, and ECF/ICF ratio in different subpopulations were demonstrated in Tale 5. The correlations between $\Sigma$ DEHP levels and BMI were more evident in subjects with older age ( $\geqq 40$ year-old), and not active smoker. The relationship between DEHP with estimated percent body fat and ECF/ICF ratio were more evident in subjects with younger age (20-39 year-old), women, and non-Hispanic white ethnic. In addition, the associations between $\mathrm{DEEHP}$ and ECF/ICF ratio were also evident in subjects who were not active smokers.

\section{Discussion}

In the current study, we reported levels of DEHP metabolites were positively correlated with parameters of body measures (weight, BMI, and waist) and parameters of BIA (fat mass, estimated percent body fat, ECF, and ECF/ICF ratio). Although the relationship between DEHP exposure and obesity parameters has been extensively studied, this is the first report identifying a positive association between urine DEHP metabolites with ECF, and ECF/ICF ratio in a nationally representative survey of U.S. adults. The representativeness of the study population is the main advantage of this study and we were able to control many covariates in the comprehensive NHANES database.

Obesity increases the incidence of cardiovascular disease, diabetes mellitus, and certain cancers [39]. In the United States, the prevalence of obesity was $42.4 \%$ in adults [40]. Its related health issues have a significant economic impact on the U.S. medical system, estimated to be approximately US $\$ 147$ billion in 2008. Traditionally, the causes of obesity is resulting from a combination of behavior, genetics, diseases and medications [41]. Recently, it has been discovered that the effects of environmental toxins, especially those defined as endocrine-disrupting chemicals (EDCs), can promote obesity and are called " obesogens " $[25,42]$. In vitro studies, the mono- and dicarboxylic acid metabolites of DEHP have affinity with the receptors of peroxisome proliferator-activated receptor (PPAR) a and PPARy [43]. PPARa is mainly involved in the regulation of gluconeogenesis, lipogenesis, and fatty acid metabolism. Activation of PPARycauses differentiation of pre-adipocyte cells into fat tissue. In the 3T3-L1 cell model, DEHP metabolites induce adipogenesis through this mechanism $[44,45]$. In animal studies, female $\mathrm{C} 3 \mathrm{H} / \mathrm{N}$ mice exposed to DEHP doses similar to environmental exposures increased food intake, body weight, and visceral fat deposits [46]. In male $\mathrm{C} 3 \mathrm{H} / \mathrm{He}$ mice, chronic exposure to DEHP may induce obesity through changes in energy homeostasis. The synergistic effect of hypothyroidism and hypothalamic leptin resistance is a possible mechanism [47].

Several recent epidemiological studies have surveyed the association between DEHP exposure and obesity in adults. All the previous studies used $\mathrm{BMI}$ /waist/weight gain as markers of obesity. In cross-sectional studies, one study observed higher MEHP levels were associated with increased obesity prevalence in NHANES 1999-2004 [26]. Another study using data from the 2007-2010 NHANES, the authors reported higher levels of MECPP, MEHHP, and IDEHP were associated with increased prevalence of obesity [25]. However, other studies found MEHP levels have inverse associations with obesity in both 1999-2002 NHANES data [29] and the Nurses' Health Study (NHS) and NHS2 cohorts [28]. By using physiologically-based pharmacokinetic model, a study reported the relationship between BMI and DEHP may be explained by higher energy intake and the consequent higher DEHP exposure [48]. In prospective analyses, MEOHP levels have positive trends with weight gain between baseline and year 3 . However, no statistically significant association was observed after 6 years [27]. In the current study, we found higher levels of DEHP metabolites were correlated with higher body measures parameters (weight, BMI, and waist). Differences between studies may be due to a variety of factors, such as race, measurement method, lifestyle, and food.

BMI and waist are generally used as screening tools for overweight or obesity with high specificity [21]. However, these two parameters are less sensitive in identifying adiposity, because they could not distinguish half of people with high body fat [49]. Some experts suggest the value of assessing body-fat 
percentage, which is more directly to measure adiposity [49]. In recent years, it has been proven that BIA is a more accurate tool for evaluate body composition and fluid status [50]. Previous studies on DEHP exposure and fat mass were limited to children. Some studies reported there was no correlation between DEHP exposure and adiposity [30,32,33]. Another report showed the associations of DEHP exposure with body fat depended on the timing of exposure [31]. In adults, there were few studies studying the association between DEHP exposure and body fat percentage. One study found no correlation between urine MEHP levels and adiposity measures in elderly women [35]. Another study enrolled participants from NHANES 1999-2006, the authors reported there was no association between MEHP and body-fat percentage but a negative correlation with lean mass among women [34]. The difference between our study and the above two studies is that we use many metabolites of DEHP as biological indicators instead of just MEHP. In this study, we found levels of MEOHP, MEHHP, MECPP, and $\Sigma$ DEHP was positively correlated with estimated fat mass, estimated percent body fat. The results provided the evidence of DEHP exposure might increase fat mass/body fat percentage in adults.

The fluid volume in human depends on age, gender, and body size and is also associated with human health. Extracellular fluid (ECF) is controlled by sodium balance and total body sodium content. Osmolality of the ECF is regulated by water intake and vasopressin secretion while the ECF volume is maintained by RAAS and some natriuretic factors, including atrial/brain natriuretic peptide [10]. In addition, growth hormone and sex hormones have been found to have a role in ECF volume $[23,51]$. Environmental chemicals which have capacity to inhibit 11ß-HSD-2. would be predicted to elevate blood pressure and expand ECF [17]. In mice, DEHP exposure have been reported to elevate blood pressure through activation of RAAS [19]. In premature infants, higher levels of postnatal DEHP exposure are correlated with increased blood pressure and hypertension. Statistical analysis showed this relationship was mediated by the cortisol-tocortisone ratio, suggesting the mechanism may be because DEHP inhibits the activity of $11 \beta-H S D-2$ and then activates the mineralocorticoid receptor. In this study, increased expression of ENaC and phosphorylated $\mathrm{Na}+-\mathrm{Cl}$ - cotransporter were also found in hypertensive infants [20]. Moreover, adipocytes produced $30 \%$ of the total blood angiotensinogen [52] and are also an important source of extra-adrenal aldosterone [22]. It is possible that DEHP induced activation of RAAS, and then contributes to sodium retention and ECF expansion [18]. Another possible mechanism is that DEHP, as an endocrine disruptive agent, have been reported to have estrogenic activity both in vitro and in vivo [53]. The high estrogen status will then increase ENaC activity via protein kinase $C \delta$ signaling in renal cortical collecting duct cells and expands ECF volume [23]. In the current study, we provide the first evidence that levels of DEHP metabolites were positively correlated with ECF, and ECF/ICF ratio. Since increased ECF may cause stress on the cardiovascular system [12], which may be one of the possible mechanisms of DEHP causing cardiovascular disease. However, this hypothesis still needs further study to clarify.

In the subgroup analysis, the correlation between $\Sigma$ DEHP and ECF/ICF ratio were more evident in subjects with women. Women are exposed to a higher concentration of phthalates than men because of the personal care products they used [54]. Furthermore, report has shown there were sex differences in the regulation of RAAS. The adrenal response to exogenous angiotensin II was significantly higher in women. It is possible that the effect of DEHP induced RAAS may be more evident in women than in men [55]. We also found that the association between $\Sigma$ DEHP and the ECF/ICF ratio was more pronounced among subjects who were not current smokers. Studies show that body composition may be altered by smoking habits [56, 57]. A possible explanation is that DEHP has a much weaker effect on body fluids than tobacco smoke. When considering the impact of DEHP on active smokers, the trend is too small to be statistically significant.

Our research has several limitations. First, causal inference is not suitable for cross-sectional research. Secondly, when DEHP is exposed, other undetected chemicals may be exposed at the same time, and the correlation found in the research is not caused by DEHP itself. Third, our study population is mainly composed of adults, so we cannot infer that this association also exists in children. Lastly, other restriction includes over-adjustments because of the relatively small number of cases.

\section{Conclusions}

Although previous reports have explored the association between DEHP exposure and obesity parameters, including BMI, waist circumference, and fat percentage in adults, we present the first report identifying a positive association of urine DEHP metabolites with body fluid status in a nationally representative survey of U.S. adults. Since DEHP exposure has become a worldwide concern, further research is necessary to determine the long-term mechanism and effects of low-dose DEHP exposure on human health.

\section{Abbreviations}

BIA

bioimpedance analysis

$\mathrm{BMI}$

body mass index

DEHP

di-(2-ethylhexyl) phthalate

ECF

extracellular fluid

EDCs

endocrine-disrupting chemicals

ICF

intracellular fluid

MECPP

mono(2-ethyl-5-carboxypentyl) phthalate

MEHHP 
mono(2-ethyl-5-hydroxyhexyl) phthalate

MEHP

mono (2-ethylhexyl) phthalate

MEOHP

mono(2-ethyl-5-oxohexyl) phthalate

NHANES

National Health and Nutrition Examination Survey

NHS

Nurses' Health Study

RAAS

renin-angiotensin-aldosterone system

\section{Declarations}

Ethics approval and consent to participate: National Center for Health Statistics Research Ethics Review Board Approval

Consent for publication: Consent documents are part of the protocol for the NHANES, conducted by the National Center for Health Statistics.

Availability of data and material: All the data are available at NHANES website.

Competing interests: The authors declared that the methods were carried out in accordance with the approved guidelines. The authors have declared that no other conflict of interest exists. No competing financial interest was declared.

Funding: There was no specific funding for this study

Author's contributions: Chien-Yu Lin developed the theoretical concept and performed the analytic calculations. Wei-Jie Wang and Chia-Sung Wang contributed to manuscript drafting. Chi-Kang Wang verified the analytical method. An-Ming Yang and Chien-Yu Lin contributed to critical discussion and the final version of the manuscript.

Acknowledgments: We would like to thank the many individuals who have contributed to the National Health and Nutrition Examination Survey data, including all of the anonymous participants in the present study. We are particularly grateful to those who carried out the laboratory assays for DEHP at the Division of Environmental Health Laboratory Sciences, National Center for Environmental Health, Centers for Disease Control and Prevention.

\section{References}

1. Liao KW, Kuo PL, Huang HB, Chang JW, Chiang HC, Huang PC: Increased risk of phthalates exposure for recurrent pregnancy loss in reproductive-aged women. Environmental pollution (Barking, Essex. 1987) 2018, 241:969-977.

2. Wang W, Leung AOW, Chu LH, Wong MH: Phthalates contamination in China: Status, trends and human exposure-with an emphasis on oral intake. Environmental pollution (Barking, Essex. 1987) 2018, 238:771-782.

3. Meeker JD, Ferguson KK. Urinary phthalate metabolites are associated with decreased serum testosterone in men, women, and children from NHANES 2011-2012. J Clin Endocrinol Metab. 2014;99(11):4346-52.

4. Hoppin JA, Brock JW, Davis BJ, Baird DD. Reproducibility of urinary phthalate metabolites in first morning urine samples. Environmental health perspectives. 2002;110(5):515-8.

5. Wang IJ, Lin CC, Lin YJ, Hsieh WS, Chen PC. Early life phthalate exposure and atopic disorders in children: a prospective birth cohort study. Environment international. 2014;62:48-54.

6. Schaedlich K, Gebauer S, Hunger L, Beier LS, Koch HM, Wabitsch M, Fischer B, Ernst J: DEHP deregulates adipokine levels and impairs fatty acid storage in human SGBS-adipocytes. Scientific reports 2018, 8(1):3447.

7. James-Todd T, Stahlhut R, Meeker JD, Powell SG, Hauser R, Huang T, Rich-Edwards J. Urinary phthalate metabolite concentrations and diabetes among women in the National Health and Nutrition Examination Survey (NHANES) 2001-2008. Environmental health perspectives 2012, 120(9):1307-1313.

8. Trasande L, Sathyanarayana S, Spanier AJ, Trachtman H, Attina TM, Urbina EM. Urinary phthalates are associated with higher blood pressure in childhood. J Pediatr. 2013;163(3):747-53 e741.

9. Edelman IS, Leibman J. Anatomy of body water and electrolytes. Am J Med. 1959;27:256-77.

10. Roumelioti ME, Glew RH, Khitan ZJ, Rondon-Berrios H, Argyropoulos CP, Malhotra D, Raj DS, Agaba El, Rohrscheib M, Murata GH, et al. Fluid balance concepts in medicine: Principles and practice. World journal of nephrology. 2018;7(1):1-28.

11. Seo HS, Kim EJ, Kim SW, Im SI, Na JO, Choi CU, Lim HE, Won Kim J, Rha SW, Park CG. Extracellular fluid adjusted for body size is contracted in hypertension. Hypertension research: official journal of the Japanese Society of Hypertension. 2013;36(10):916-21.

12. Park S, Lee CJ, Jhee JH, Yun HR, Kim H, Jung SY, Kee YK, Yoon CY, Park JT, Kim HC, et al: Extracellular Fluid Excess Is Significantly Associated With Coronary Artery Calcification in Patients With Chronic Kidney Disease. Journal of the American Heart Association 2018, 7(13).

13. Lee JY, Ryu HS, Yoon SS, Kim EH, Yoon SW. Extracellular-to-Intracellular Fluid Volume Ratio as a Prognostic Factor for Survival in Patients With Metastatic Cancer. Integrative cancer therapies. 2019;18:1534735419847285.

Page $11 / 13$ 
14. Chen W, Guo LJ, Wang T. Extracellular water/intracellular water is a strong predictor of patient survival in incident peritoneal dialysis patients. Blood purification. 2007;25(3):260-6.

15. Kim EJ, Choi MJ, Lee JH, Oh JE, Seo JW, Lee YK, Yoon JW, Kim HJ, Noh JW, Koo JR. Extracellular Fluid/Intracellular Fluid Volume Ratio as a Novel Risk Indicator for All-Cause Mortality and Cardiovascular Disease in Hemodialysis Patients. PloS one. 2017;12(1):e0170272.

16. Lin Y-P, Yu W-C, Hsu T-L, Ding PY-A, Yang W-C, Chen C-H. The extracellular fluid-to-intracellular fluid volume ratio is associated with large-artery structure and function in hemodialysis patients. Am J Kidney Dis. 2003;42(5):990-9.

17. Kirkley AG, Sargis RM. Environmental endocrine disruption of energy metabolism and cardiovascular risk. Curr Diabetes Rep. 2014;14(6):494.

18. Harrison-Bernard LM. The renal renin-angiotensin system. Adv Physiol Educ. 2009;33(4):270-4.

19. Xie X, Deng T, Duan J, Ding S, Yuan J, Chen M. Comparing the effects of diethylhexyl phthalate and dibutyl phthalate exposure on hypertension in mice. Ecotoxicol Environ Saf. 2019;174:75-82.

20. Jenkins R, Tackitt S, Gievers L, Iragorri S, Sage K, Cornwall T, O'Riordan D, Merchant J, Rozansky D. Phthalate-associated hypertension in premature infants: a prospective mechanistic cohort study. Pediatr Nephrol (Berlin Germany). 2019;34(8):1413-24.

21. Ribeiro C, Mendes V, Peleteiro B, Delgado I, Araújo J, Aggerbeck M, Annesi-Maesano I, Sarigiannis D, Ramos E. Association between the exposure to phthalates and adiposity: A meta-analysis in children and adults. Environmental research. 2019;179(Pt A):108780.

22. Briones AM, Nguyen Dinh Cat A, Callera GE, Yogi A, Burger D, He Y, Corrêa JW, Gagnon AM, Gomez-Sanchez CE, Gomez-Sanchez EP, et al: Adipocytes produce aldosterone through calcineurin-dependent signaling pathways: implications in diabetes mellitus-associated obesity and vascular dysfunction. Hypertension (Dallas, Tex. 1979) 2012, 59(5):1069-1078.

23. Yusef YR, Thomas W, Harvey BJ. Estrogen increases ENaC activity via PKCS signaling in renal cortical collecting duct cells. Physiological reports 2014, 2(5).

24. Khalil SF, Mohktar MS, Ibrahim F. The Theory and Fundamentals of Bioimpedance Analysis in Clinical Status Monitoring and Diagnosis of Diseases. Sensors. 2014;14(6):10895-928.

25. Buser MC, Murray HE, Scinicariello F. Age and sex differences in childhood and adulthood obesity association with phthalates: analyses of NHANES 2007-2010. Int J Hyg Environ Health. 2014;217(6):687-94.

26. Yaghjyan L, Sites S, Ruan Y, Chang SH. Associations of urinary phthalates with body mass index, waist circumference and serum lipids among females: National Health and Nutrition Examination Survey 1999-2004. International journal of obesity (2005) 2015, 39(6):994-1000.

27. Díaz Santana MV, Hankinson SE, Bigelow C, Sturgeon SR, Zoeller RT, Tinker L, Manson JAE, Calafat AM, Meliker JR, Reeves KW. Urinary concentrations of phthalate biomarkers and weight change among postmenopausal women: a prospective cohort study. Environmental health: a global access science source. 2019;18(1):20.

28. Song Y, Hauser R, Hu FB, Franke AA, Liu S, Sun Q. Urinary concentrations of bisphenol A and phthalate metabolites and weight change: a prospective investigation in US women. Int J Obes. 2014;38(12):1532-7.

29. Hatch EE, Nelson JW, Qureshi MM, Weinberg J, Moore LL, Singer M, Webster TF. Association of urinary phthalate metabolite concentrations with body mass index and waist circumference: a cross-sectional study of NHANES data, 1999-2002. Environmental health: a global access science source 2008, 7:27.

30. Buckley JP, Engel SM, Mendez MA, Richardson DB, Daniels JL, Calafat AM, Wolff MS, Herring AH. Prenatal Phthalate Exposures and Childhood Fat Mass in a New York City Cohort. Environmental health perspectives. 2016;124(4):507-13.

31. Shoaff J, Papandonatos GD, Calafat AM, Ye X, Chen A, Lanphear BP, Yolton K, Braun JM: Early-Life Phthalate Exposure and Adiposity at 8 Years of Age. Environmental health perspectives 2017, 125(9):097008.

32. Maresca MM, Hoepner LA, Hassoun A, Oberfield SE, Mooney SJ, Calafat AM, Ramirez J, Freyer G, Perera FP, Whyatt RM, et al. Prenatal Exposure to Phthalates and Childhood Body Size in an Urban Cohort. Environmental health perspectives. 2016;124(4):514-20.

33. Chang CH, Chen CF, Tsai YA, Wang SL, Huang PC, Chen BH, Wu MT, Chen CC, Hsiung CA, Chen ML. The sex-specific association of phthalate exposure with DNA methylation and characteristics of body fat in children. Sci Total Environ. 2020;737:139833.

34. Corbasson I, Hankinson SE, Stanek EJ 3rd, Reeves KW. Urinary bisphenol-A, phthalate metabolites and body composition in US adults, NHANES 19992006. International journal of environmental health research 2016, 26(5-6):606-617.

35. Lind PM, Roos V, Rönn M, Johansson L, Ahlström H, Kullberg J, Lind L. Serum concentrations of phthalate metabolites are related to abdominal fat distribution two years later in elderly women. Environmental health: a global access science source. 2012;11:21.

36. NHANES. 2003-2004 http://wwwn.cdc.gov/Nchs/Nhanes/Search/nhanes03_04.aspx.

37. Braun JM, Smith KW, Williams PL, Calafat AM, Berry K, Ehrlich S, Hauser R. Variability of urinary phthalate metabolite and bisphenol A concentrations before and during pregnancy. Environmental health perspectives. 2012;120(5):739-45.

38. ANALYTIC. AND REPORTING GUIDELINES: The National Health and Nutrition Examination Survey (NHANES) http://www.cdc.gov/nchs/data/nhanes/nhanes_03_04/nhanes_analytic_guidelines_dec_2005.pdf.

39. Li TY, Rana JS, Manson JE, Willett WC, Stampfer MJ, Colditz GA, Rexrode KM, Hu FB. Obesity as compared with physical activity in predicting risk of coronary heart disease in women. Circulation. 2006;113(4):499-506.

40. Prevalence of obesity and severe obesity among adults: United States, 2017-2018. In. Edited by National Center for Health Statistics. Division of H, Nutrition Examination S. Hyattsville, MD; 2020.

41. Adult obesity causes \& consequences https://www.cdc.gov/obesity/adult/causes.html.

Page $12 / 13$ 
42. Grün F, Blumberg B. Environmental obesogens: organotins and endocrine disruption via nuclear receptor signaling. Endocrinology. 2006;147(6 Suppl):505.

43. Ito Y, Kamijima M, Nakajima T. Di(2-ethylhexyl) phthalate-induced toxicity and peroxisome proliferator-activated receptor alpha: a review. Environ Health Prev Med. 2019;24(1):47.

44. Feige JN, Gelman L, Rossi D, Zoete V, Métivier R, Tudor C, Anghel SI, Grosdidier A, Lathion C, Engelborghs Y, et al. The Endocrine Disruptor Monoethylhexyl-phthalate Is a Selective Peroxisome Proliferator-activated Receptor Y Modulator That Promotes Adipogenesis*. J Biol Chem. 2007;282(26):1915266.

45. Hurst CH, Waxman DJ. Activation of PPARalpha and PPARgamma by environmental phthalate monoesters. Toxicol Sci. 2003;74(2):297-308.

46. Schmidt JS, Schaedlich K, Fiandanese N, Pocar P, Fischer B. Effects of di(2-ethylhexyl) phthalate (DEHP) on female fertility and adipogenesis in C3H/N mice. Environmental health perspectives. 2012;120(8):1123-9.

47. Lv Z, Cheng J, Huang S, Zhang Y, Wu S, Qiu Y, Geng Y, Zhang Q, Huang G, Ma Q, et al. DEHP induces obesity and hypothyroidism through both central and peripheral pathways in C3H/He mice. Obesity (Silver Spring Md). 2016;24(2):368-78.

48. Campbell JL Jr, Yoon M, Ward PL, Fromme H, Kessler W, Phillips MB, Anderson WA, Clewell HJ 3rd, Longnecker MP. Excretion of Di-2-ethylhexyl phthalate (DEHP) metabolites in urine is related to body mass index because of higher energy intake in the overweight and obese. Environment international. 2018;113:91-9.

49. Okorodudu DO, Jumean MF, Montori VM, Romero-Corral A, Somers VK, Erwin PJ, Lopez-Jimenez F: Diagnostic performance of body mass index to identify obesity as defined by body adiposity: a systematic review and meta-analysis. International journal of obesity (2005) 2010, 34(5):791-799.

50. de-Mateo-Silleras B, de-la-Cruz-Marcos S, Alonso-Izquierdo L, Camina-Martín MA, Marugán-de-Miguelsanz JM. Redondo-Del-Río MP: Bioelectrical impedance vector analysis in obese and overweight children. PloS one. 2019;14(1):e0211148.

51. Johannsson G, Gibney J, Wolthers T, Leung KC, Ho KK. Independent and combined effects of testosterone and growth hormone on extracellular water in hypopituitary men. J Clin Endocrinol Metab. 2005;90(7):3989-94.

52. Padilla J, Vieira-Potter VJ, Jia G, Sowers JR. Role of perivascular adipose tissue on vascular reactive oxygen species in type 2 diabetes: a give-and-take relationship. Diabetes. 2015;64(6):1904-6.

53. Chen X, Xu S, Tan T, Lee ST, Cheng SH, Lee FW, Xu SJ, Ho KC. Toxicity and estrogenic endocrine disrupting activity of phthalates and their mixtures. Int J Environ Res Public Health. 2014;11(3):3156-68.

54. Parlett LE, Calafat AM, Swan SH. Women's exposure to phthalates in relation to use of personal care products. J Expo Sci Environ Epidemiol. 2013;23(2):197-206.

55. Toering TJ, Gant CM, Visser FW, van der Graaf AM, Laverman GD, Danser AHJ, Faas MM, Navis G, Lely AT. Sex differences in renin-angiotensinaldosterone system affect extracellular volume in healthy subjects. American journal of physiology Renal physiology. 2018;314(5):F873-f878.

56. Kleppinger A, Litt MD, Kenny AM, Oncken CA. Effects of smoking cessation on body composition in postmenopausal women. J Womens Health (Larchmt). 2010;19(9):1651-7.

57. Efendi V, Özalevli S, Naz I, Kılınç O. The effects of smoking on body composition, pulmonary function, physical activity and health-related quality of life among healthy women. Tuberkuloz ve toraks. 2018;66(2):101-8.

\section{Supplementary Files}

This is a list of supplementary files associated with this preprint. Click to download.

- Supplementalsection.docx 